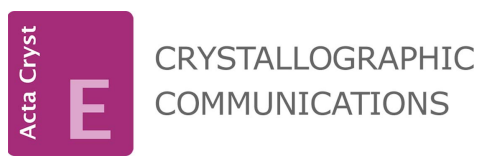

ISSN 2056-9890

Received 15 December 2014

Accepted 18 January 2015

Edited by M. Weil, Vienna University of

Technology, Austria

Keywords: crystal structure; barbiturate;

hydrogen bonding; anaesthetic

CCDC reference: 1044166

Supporting information: this article has

supporting information at journals.iucr.org/e

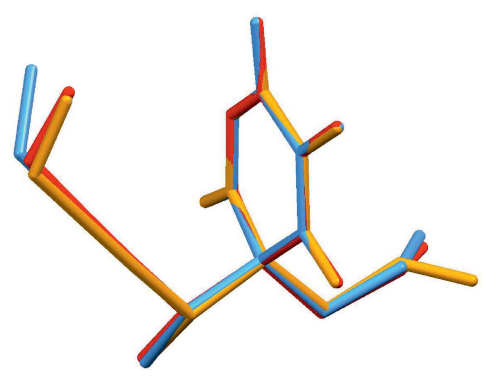

OPEN $\odot$ ACCESS

\section{Crystal structure of the $\alpha$-racemate of methohexital}

\author{
Thomas Gelbrich* and Ulrich J. Griesser
}

Institute of Pharmacy, University of Innsbruck, Innrain 52c, 6020 Innsbruck, Austria. *Correspondence e-mail: thomas.gelbrich@uibk.ac.at

Molecules of the title compound, $\mathrm{C}_{14} \mathrm{H}_{18} \mathrm{~N}_{2} \mathrm{O}_{3}$ [systematic name: 5-allyl-5-(hex3-yn-2-yl)-1-methylpyrimidine-2,4,6(1H,3H,5H)-trione in the $\left(R_{b} S_{h}\right) /\left(S_{b} R_{h}\right)$ racemic form], are connected by mutual $\mathrm{N}-\mathrm{H} \cdots \mathrm{O}=\mathrm{C}$ hydrogen bonds in which the carbonyl group at the 2-position of the pyrimidinetrione ring is employed. These interactions result in an inversion dimer which displays a central $R_{2}^{2}(8)$ ring motif. This dimer is topologically distinct from that of the previously reported $\left(S_{b} R_{h}\right)$ form, which is, however, also based on an $R_{2}^{2}(8)$ motif. The methyl group at the 1-position of the pyrimidinetrione ring in the title structure is disordered over two sets of sites in a 0.57 (2):0.43 (2) ratio.

\section{Chemical context}

The title compound is a barbiturate derivative, the Na salt of which (trade name Brevimytal, Eli Lilly) is a widely used short-acting anaesthetic with a rapid onset of action. The molecule contains two asymmetric centres and can exist as two diastereomeric enantiomer pairs. Its stereoisomerism is known to affect the anaesthetic activity and possible side effects of the drug (Gibson et al., 1959). The crystal structure of the $\left(S_{b} R_{h}\right)$ form of methohexital was previously reported by Brunner et al. (2003), who also established that the commercial product ( $\alpha$-racemate) consists of the $\left(R_{b} S_{h}\right)$ and $\left(S_{b} R_{h}\right)$ isomers.

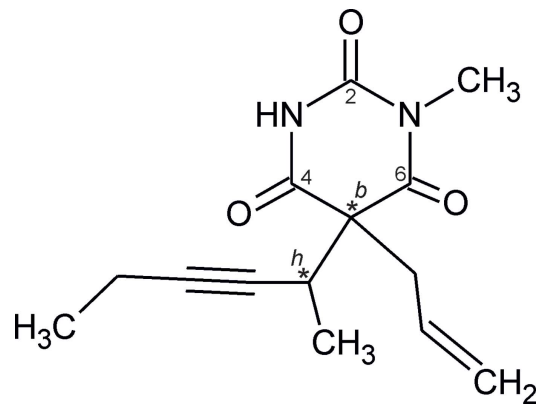

\section{Structural commentary}

This study confirmed the presence of the $\left(R_{b} S_{h}\right) /\left(S_{b} R_{h}\right)$ racemate. The molecule (Fig. 1) displays an approximately planar pyrimidinetrione unit in which the oxygen atoms of the $\mathrm{C} 2$ and C4 carbonyl groups lie at distances of -0.160 (2) and 0.156 (2) $\AA$ from the mean plane of the six-membered ring (r.m.s. deviation $=0.046 \AA$ ). The conformation of the two 5substituents of the ring is characterized by three parameters, the torsion angles $\mathrm{C} 5-\mathrm{C} 7-\mathrm{C} 8-\mathrm{C} 9$ of -103.3 (2) and $\mathrm{C} 10-$ $\mathrm{C} 5-\mathrm{C} 7-\mathrm{C} 8$ of $-171.51(13)^{\circ}$ and the pseudo-torsion angle $\mathrm{C} 5-\mathrm{C} 10 \cdots \mathrm{C} 13-\mathrm{C} 14$ of $23.2(2)^{\circ}$.

The previously reported $\left(S_{b} R_{h}\right)$ form contains two independent molecules (denoted $A$ and $B$ ), which differ from the 


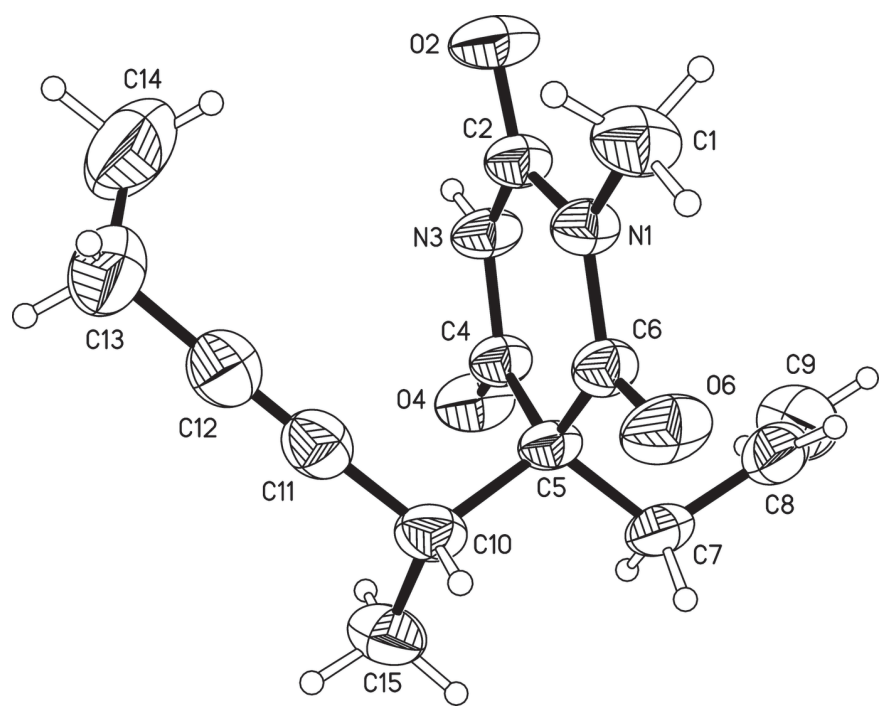

Figure 1

The molecular structure of the title compound with displacement ellipsoids drawn at the $50 \%$ probability level; hydrogen atoms are drawn as spheres of arbitrary size.

molecule of the title structure in the conformation adopted by the terminal groups of both 5-substituents (Fig. 2). Specifically, in molecule $A$, the torsion angle analogous to $\mathrm{C} 5-\mathrm{C} 7-\mathrm{C} 8-$ C9 in the present $\alpha$-racemate is $125.3^{\circ}$, and the pseudo-torsion angles analogous to $\mathrm{C} 5-\mathrm{C} 10 \cdots \mathrm{C} 13-\mathrm{C} 14$ of the title structure are $-15.4^{\circ}(A)$ and $-26.3^{\circ}(B)$.

\section{Supramolecular features}

Two molecules are linked to one another by two mutual antiparallel $\mathrm{N}-\mathrm{H} \cdots \mathrm{O}=\mathrm{C}$ bonds so that an inversion dimer is formed (Table 1, Fig. 3), which displays a central $R_{2}^{2}(8)$ ring motif (Etter et al., 1990; Bernstein et al., 1995). This interaction involves the carbonyl group at the 2-position of the ring. The

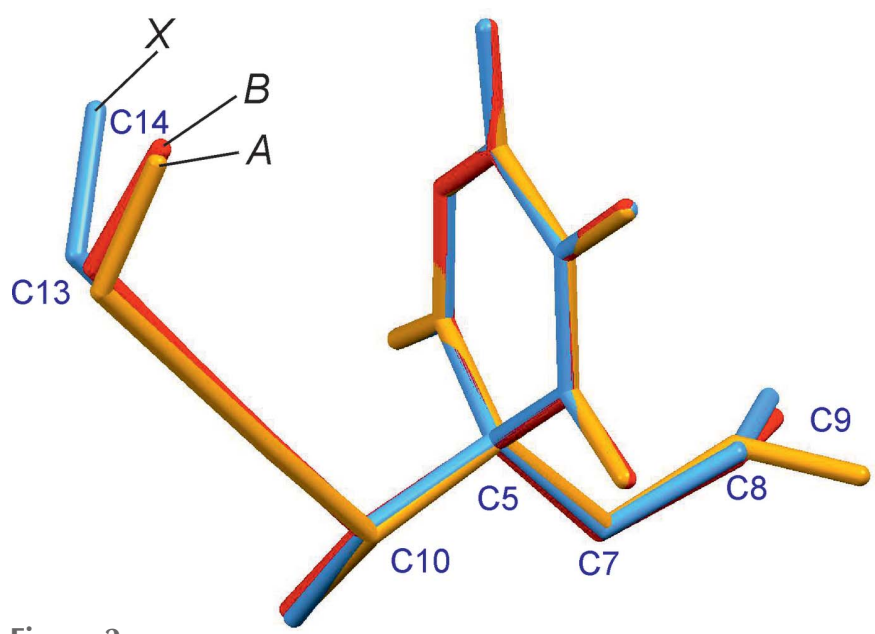

Figure 2

Overlay of the molecule of the $\alpha$-racemate (denoted $X$ ) with the two independent molecules $(A, B)$ of the previously reported $\left(S_{b} R_{h}\right)$ form, generated by least-squares fits of their 1-methyl-2,4,6-pyrimidinetrione units (ten non-H atomic positions).
Table 1

Hydrogen-bond geometry $\left(\AA,^{\circ}\right)$.

\begin{tabular}{lllll}
\hline$D-\mathrm{H} \cdots A$ & $D-\mathrm{H}$ & $\mathrm{H} \cdots A$ & $D \cdots A$ & $D-\mathrm{H} \cdots A$ \\
\hline $\mathrm{N} 3-\mathrm{H} 3 \cdots \mathrm{O} 2^{\mathrm{i}}$ & $0.85(2)$ & $2.03(2)$ & $2.8826(17)$ & $173.2(17)$ \\
\hline
\end{tabular}

Symmetry code: (i) $-x,-y,-z+1$.

$R_{2}^{2}(8)$ ring motif is also present in the $\left(S_{b} R_{h}\right)$ form (Brunner et al., 2003) where it connects the two crystallographically independent molecules. However, in this case the dimer is based on two topologically distinct $\mathrm{N}-\mathrm{H} \cdots \mathrm{O}=\mathrm{C}$ interactions which involve the carbonyl groups at the 4-position of the ring of molecule $A$ and at the 2-position of molecule $B$.

\section{Database survey}

The Cambridge Structural Database (Groom \& Allen, 2014; Version 3.35) contains 11 unique entries for derivatives of barbituric acid which are analogous to the title compound and substituted at the 1-position, but not at the 3-position of the six-membered ring. A common characteristic of these compounds is the presence of one hydrogen-bond donor group $(\mathrm{NH})$ and three potential acceptor groups, viz. the carbonyl groups at the ring positions 2, 4 and 6 . Thus, three topologically distinct hydrogen-bonding acceptor interactions are possible. Additionally, there is a competition between possible dimer and catemer motifs, which is similar to the competition between hydrogen-bonded dimer and catemer motifs between carboxyl groups (Beyer \& Price; 2000) or carboxamide groups (Arlin et al., 2010, 2011).

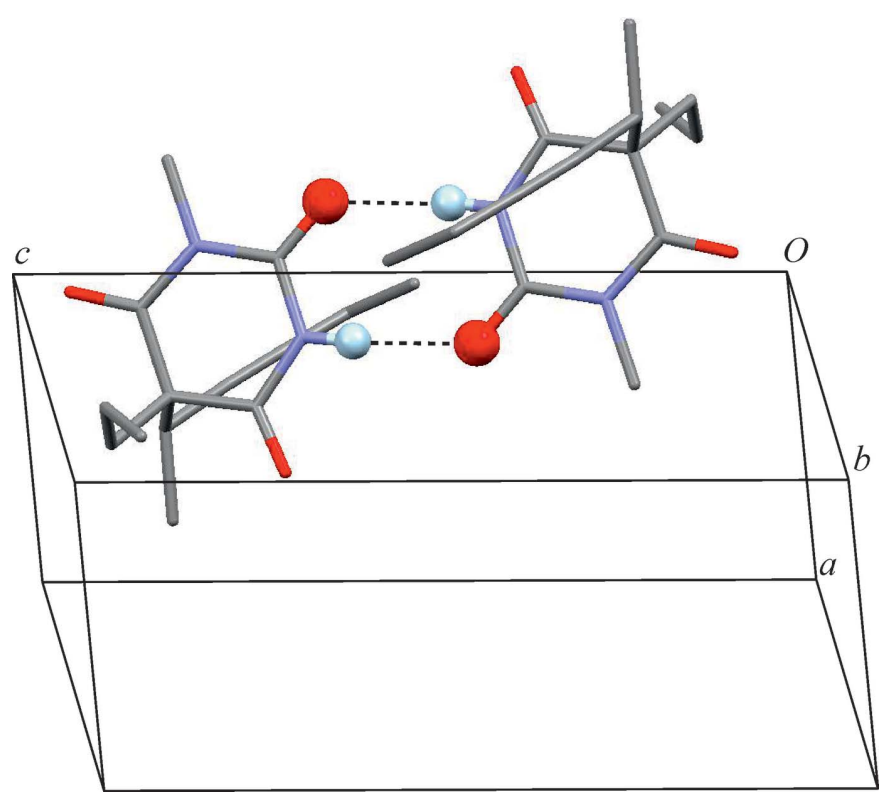

Figure 3

The $\mathrm{N}-\mathrm{H} \cdots \mathrm{O}=\mathrm{C}$ hydrogen-bonded inversion dimer displaying a central $R_{2}^{2}(8)$ ring. These interactions (dotted lines) involve the carbonyl group at the 2-position of the six-membered ring. $\mathrm{O}$ and $\mathrm{H}$ atoms engaged in hydrogen bonding are drawn as spheres. 
<smiles>[R]N1C(=O)N([Tl])C(=O)C([R])([R])C1=O</smiles>

2-position dimer: 3 catemer: 1

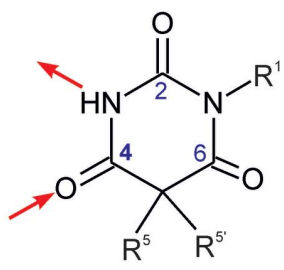

4-position dimer: 2 catemer: 0

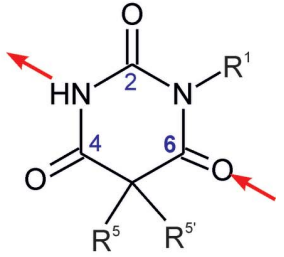

6-position

dimer: 0 catemer: 5
Figure 4

The three fundamental connection modes for the formation of $\mathrm{N}-$ $\mathrm{H} \cdots \mathrm{O}=\mathrm{C}$ bonds in 1-substituted derivatives of barbituric acid arising from the involvement of different carbonyl groups, and the corresponding numbers of observed dimer and catemer isomers. The $\left(S_{b} R_{h}\right)$ form of methohexithal contains a dimer with mixed $\mathrm{N}-\mathrm{H} \cdots \mathrm{O}=\mathrm{C} 2 / \mathrm{N}-$ $\mathrm{H} \cdots \mathrm{O}=\mathrm{C} 4$ connectivity and was therefore not included.

Closer inspection of the geometric possibilities (Fig. 4) shows that dimer formation is feasible for $\mathrm{N}-\mathrm{H} \cdots \mathrm{O}=\mathrm{C} 2$ and $\mathrm{N}-\mathrm{H} \cdots \mathrm{O}=\mathrm{C} 4$ connections only, whereas $\mathrm{N}-\mathrm{H} \cdots \mathrm{O}=\mathrm{C} 6$ should be the preferred connection mode for chain formation. Indeed, five crystal structures containing $\mathrm{N}-\mathrm{H} \cdots \mathrm{O}=\mathrm{C} 6$ chain motifs are known and their CSD refcodes are DMCYBA01 (Nichol \& Clegg, 2005), DULMED (Gelbrich et al., 2010), MDEBAR (Wunderlich, 1973), MIBABA (Wilhelm \& Fischer, 1976), OBIPUM (Gelbrich \& Griesser, 2009). So far, the crystal structure with refcode VEMQUB (Savechenkov et al., 2012) is the only example in the set where another chain type, viz. $\mathrm{N}-\mathrm{H} \cdots \mathrm{O}=\mathrm{C} 2$, is present.

Apart from the title structure, two analogues with refcodes CXALBA (Dideberg et al., 1975) and DULMAZ (Gelbrich et al., 2010) also form $\mathrm{N}-\mathrm{H} \cdots \mathrm{O}=\mathrm{C} 2$ bonded dimers. The alternative $\mathrm{N}-\mathrm{H} \cdots \mathrm{O}=\mathrm{C} 4$ dimer was observed in the two structures with refcodes ALLBTC (Pyżalska et al., 1980) and MEPBAB01 (Lewis et al., 2005). The $\left(S_{b} R_{h}\right)$ form of methohexital provides the only case of a dimer based on a mixed $\mathrm{N}-$ $\mathrm{H} \cdots \mathrm{O}=\mathrm{C} 2 / \mathrm{N}-\mathrm{H} \cdots \mathrm{O}=\mathrm{C} 4$ connectivity.

\section{Synthesis and crystallization}

The crystals investigated in this study were obtained at room temperature, by slow evaporation from an aqueous solution of the $\alpha$-racemate of methohexital (Lilly Research Centre Ltd., Windlesham, England).

\section{Refinement}

Crystal data, data collection and structure refinement details are summarized in Table 2. $\mathrm{H}$ atoms were identified in difference maps. The $\mathrm{H}$ atoms of the $\mathrm{C} 14$ methyl group and disordered C1 methyl group [occupancy ratio 0.57 (2):0.43 (2)] were idealized and included as rigid groups allowed to rotate but not tip $(\mathrm{C}-\mathrm{H}=0.96 \AA)$ and refined with $U_{\text {iso }}$ set to $1.5 U_{\text {eq }}(\mathrm{C})$ of the parent carbon atom. $\mathrm{H}$ atoms bonded to secondary $\mathrm{CH}_{2}(\mathrm{C}-\mathrm{H}=0.97 \AA)$, tertiary $\mathrm{CH}(\mathrm{C}-\mathrm{H}=0.98 \AA)$
Table 2

Experimental details.

Crystal data

Chemical formula

$M_{\mathrm{r}}$

Crystal system, space group

Temperature (K)

$a, b, c(\AA)$

$\alpha, \beta, \gamma\left({ }^{\circ}\right)$

$V\left(\AA^{3}\right)$

Z

Radiation type

$\mu\left(\mathrm{mm}^{-1}\right)$

Crystal size (mm)

$\mathrm{C}_{14} \mathrm{H}_{18} \mathrm{~N}_{2} \mathrm{O}_{3}$

262.30

Triclinic, $P \overline{1}$

293

7.7502 (6), 7.9792 (5), 12.6881 (10)

93.713 (6), 96.226 (6), 113.314 (7)

$711.32(10)$

2

Mo $K \alpha$

0.09

$0.35 \times 0.20 \times 0.20$

Data collection

Diffractometer

Absorption correction

$T_{\min }, T_{\max }$

No. of measured, independent and observed $[I>2 \sigma(I)]$ reflections

$R_{\text {int }}$

$(\sin \theta / \lambda)_{\max }\left(\AA^{-1}\right)$

Agilent Xcalibur (Ruby, Gemini ultra)

Multi-scan (CrysAlis PRO;

Agilent, 2012)

$0.883,1.000$

$6896,3363,2462$

0.022

0.690

Refinement

$R\left[F^{2}>2 \sigma\left(F^{2}\right)\right], w R\left(F^{2}\right), S$

No. of reflections

No. of parameters

$\mathrm{H}$-atom treatment

$0.049,0.135,1.05$

3363

180

$\mathrm{H}$ atoms treated by a mixture of independent and constrained refinement

$\Delta \rho_{\max }, \Delta \rho_{\min }\left(\mathrm{e} \AA^{-3}\right)$ $0.22,-0.20$

Computer programs: CrysAlis PRO (Agilent, 2012), SHELXS97 (Sheldrick, 2008), SHELXL2014/6 (Sheldrick, 2015), XP in SHELXTL (Sheldrick, 2008), Mercury (Macrae et al., 2006) and publCIF (Westrip, 2010).

carbon and aromatic $\mathrm{CH}$ carbon atoms $(\mathrm{C}-\mathrm{H}=0.93 \AA)$ were positioned geometrically and refined with $U_{\text {iso }}$ set to $1.2 U_{\text {eq }}(\mathrm{C})$ of the parent carbon atom. The $\mathrm{NH}$ hydrogen atom was refined with a restrained distance $[\mathrm{N}-\mathrm{H}=0.86(2) \AA]$ and its $U_{\text {iso }}$ parameter was freely refined.

\section{Acknowledgements}

We thank Volker Kahlenberg for access to the X-ray diffraction instrument used in this study.

\section{References}

Agilent (2012). CrysAlis PRO. Agilent Technologies, Yarnton, England.

Arlin, J.-B., Johnston, A., Miller, G. J., Kennedy, A. R., Price, S. L. \& Florence, A. J. (2010). CrystEngComm 12, 64-66.

Arlin, J.-B., Price, L. S., Price, S. L. \& Florence, A. J. (2011). Chem. Commun. 47, 7074-7076.

Bernstein, J., Davis, R. E., Shimoni, L. \& Chang, N.-L. (1995). Angew. Chem. Int. Ed. Engl. 34, 1555-1573.

Beyer, T. \& Price, S. L. (2000). J. Phys. Chem. B, 104, 2647-2655.

Brunner, H., Ittner, K.-P., Lunz, D., Schmatloch, S., Schmidt, T. \& Zabel, M. (2003). Eur. J. Org. Chem. pp. 855-862.

Dideberg, O., Dupont, L. \& Pyzalska, D. (1975). Acta Cryst. B31, 685688.

Etter, M. C., MacDonald, J. C. \& Bernstein, J. (1990). Acta Cryst. B46, 256-262. 
Gelbrich, T. \& Griesser, U. (2009). Private communication (refcode OBIPUM). CCDC, Cambridge, England.

Gelbrich, T., Zencirci, N. \& Griesser, U. J. (2010). Acta Cryst. C66, o55-o58.

Gibson, W. R., Doran, W. J., Wood, W. C. \& Swanson, E. E. (1959). J. Pharmacol. Exp. Ther. 125, 23-27.

Groom, C. R. \& Allen, F. H. (2014). Angew. Chem. Int. Ed. 53, 115121.

Lewis, W., McKeown, R. H. \& Robinson, W. T. (2005). Acta Cryst. E61, o799-o800.

Macrae, C. F., Edgington, P. R., McCabe, P., Pidcock, E., Shields, G. P., Taylor, R., Towler, M. \& van de Streek, J. (2006). J. Appl. Cryst. 39, 453-457.
Nichol, G. S. \& Clegg, W. (2005). Acta Cryst. E61, o1004-o1006.

Pyżalska, D., Pyżalski, R. \& Borowiak, T. (1980). Acta Cryst. B36, 1672-1675.

Savechenkov, P. Y., Zhang, X., Chiara, D. C., Stewart, D. S., Ge, R., Zhou, X., Raines, D. E., Cohen, J. B., Forman, S. A., Miller, K. W. \& Bruzik, K. S. (2012). J. Med. Chem. 55, 6554-6565.

Sheldrick, G. M. (2008). Acta Cryst. A64, 112-122.

Sheldrick, G. M. (2015). Acta Cryst. C71, 3-8.

Westrip, S. P. (2010). J. Appl. Cryst. 43, 920-925.

Wilhelm, E. \& Fischer, K. F. (1976). Cryst. Struct. Commun. 5, 507510.

Wunderlich, H. (1973). Acta Cryst. B29, 168-173. 


\section{supporting information}

Acta Cryst. (2015). E71, 206-209 [doi:10.1107/S205698901500105X]

\section{Crystal structure of the $\alpha$-racemate of methohexital}

\section{Thomas Gelbrich and Ulrich J. Griesser}

\section{Computing details}

Data collection: CrysAlis PRO (Agilent, 2012); cell refinement: CrysAlis PRO (Agilent, 2012); data reduction: CrysAlis PRO (Agilent, 2012); program(s) used to solve structure: SHELXS97 (Sheldrick, 2008); program(s) used to refine structure: SHELXL2014/6 (Sheldrick, 2015); molecular graphics: XP in SHELXTL (Sheldrick, 2008) and Mercury (Macrae et al., 2006); software used to prepare material for publication: publCIF (Westrip, 2010).

\section{5-Allyl-5-(hex-3-yn-2-yl)-1-methylpyrimidine-2,4,6(1H,3H,5H)-trione}

Crystal data

$\mathrm{C}_{14} \mathrm{H}_{18} \mathrm{~N}_{2} \mathrm{O}_{3}$

$M_{r}=262.30$

Triclinic, $P \overline{1}$

$a=7.7502(6) \AA$

$b=7.9792(5) \AA$

$c=12.6881(10) \AA$

$\alpha=93.713(6)^{\circ}$

$\beta=96.226(6)^{\circ}$

$\gamma=113.314(7)^{\circ}$

$V=711.32(10) \AA^{3}$

Data collection

Agilent Xcalibur (Ruby, Gemini ultra) diffractometer

Radiation source: Enhance (Mo) X-ray Source

Graphite monochromator

Detector resolution: 10.3575 pixels $\mathrm{mm}^{-1}$

$\omega$ scans

Absorption correction: multi-scan

(CrysAlis PRO; Agilent, 2012)

$T_{\min }=0.883, T_{\max }=1.000$

Refinement

Refinement on $F^{2}$

Least-squares matrix: full

$R\left[F^{2}>2 \sigma\left(F^{2}\right)\right]=0.049$

$w R\left(F^{2}\right)=0.135$

$S=1.05$

3363 reflections

180 parameters

0 restraints
$Z=2$

$F(000)=280$

$D_{\mathrm{x}}=1.225 \mathrm{Mg} \mathrm{m}^{-3}$

Mo $K \alpha$ radiation, $\lambda=0.71073 \AA$

Cell parameters from 1814 reflections

$\theta=4.4-28.8^{\circ}$

$\mu=0.09 \mathrm{~mm}^{-1}$

$T=293 \mathrm{~K}$

Prism, colourless

$0.35 \times 0.20 \times 0.20 \mathrm{~mm}$

6896 measured reflections

3363 independent reflections

2462 reflections with $I>2 \sigma(I)$

$R_{\text {int }}=0.022$

$\theta_{\text {max }}=29.4^{\circ}, \theta_{\min }=2.8^{\circ}$

$h=-9 \rightarrow 10$

$k=-11 \rightarrow 9$

$l=-16 \rightarrow 15$

Hydrogen site location: mixed

$\mathrm{H}$ atoms treated by a mixture of independent and constrained refinement

$w=1 /\left[\sigma^{2}\left(F_{\mathrm{o}}^{2}\right)+(0.0572 P)^{2}+0.1234 P\right]$

where $P=\left(F_{\mathrm{o}}^{2}+2 F_{\mathrm{c}}{ }^{2}\right) / 3$

$(\Delta / \sigma)_{\max }<0.001$

$\Delta \rho_{\max }=0.22 \mathrm{e} \AA^{-3}$

$\Delta \rho_{\min }=-0.20 \mathrm{e} \AA^{-3}$ 


\section{Special details}

Geometry. All e.s.d.'s (except the e.s.d. in the dihedral angle between two 1.s. planes) are estimated using the full covariance matrix. The cell e.s.d.'s are taken into account individually in the estimation of e.s.d.'s in distances, angles and torsion angles; correlations between e.s.d.'s in cell parameters are only used when they are defined by crystal symmetry. An approximate (isotropic) treatment of cell e.s.d.'s is used for estimating e.s.d.'s involving 1.s. planes.

Refinement. The $\mathrm{C} 1$ methyl group is disordered over two positions.

Fractional atomic coordinates and isotropic or equivalent isotropic displacement parameters $\left(\AA^{2}\right)$

\begin{tabular}{|c|c|c|c|c|c|}
\hline & $x$ & $y$ & $z$ & $U_{\text {iso }} * / U_{\text {eq }}$ & Occ. $(<1)$ \\
\hline N1 & $-0.00769(16)$ & -0.12155 (16) & $0.75511(9)$ & $0.0382(3)$ & \\
\hline N3 & $0.15035(17)$ & $0.08665(17)$ & $0.63981(10)$ & $0.0412(3)$ & \\
\hline $\mathrm{H} 3$ & $0.145(2)$ & $0.115(2)$ & $0.5763(16)$ & $0.055(5)^{*}$ & \\
\hline $\mathrm{O} 2$ & $-0.12784(15)$ & $-0.15309(16)$ & $0.58085(9)$ & $0.0582(4)$ & \\
\hline $\mathrm{O} 4$ & $0.42793(15)$ & $0.32706(15)$ & $0.69135(9)$ & $0.0546(3)$ & \\
\hline O6 & $0.12730(17)$ & $-0.09825(17)$ & $0.92461(9)$ & $0.0589(3)$ & \\
\hline $\mathrm{C} 1$ & $-0.1759(2)$ & $-0.2810(2)$ & $0.77286(14)$ & $0.0550(4)$ & \\
\hline H1A & -0.1624 & -0.3036 & 0.8461 & $0.082 *$ & $0.43(2)$ \\
\hline H1B & -0.2871 & -0.2566 & 0.7570 & $0.082 *$ & $0.43(2)$ \\
\hline $\mathrm{H} 1 \mathrm{C}$ & -0.1881 & -0.3870 & 0.7272 & $0.082 *$ & $0.43(2)$ \\
\hline H1D & -0.2627 & -0.3279 & 0.7074 & $0.082^{*}$ & $0.57(2)$ \\
\hline $\mathrm{H} 1 \mathrm{E}$ & -0.1380 & -0.3749 & 0.7965 & $0.082 *$ & $0.57(2)$ \\
\hline $\mathrm{H} 1 \mathrm{~F}$ & -0.2370 & -0.2445 & 0.8263 & $0.082 *$ & $0.57(2)$ \\
\hline $\mathrm{C} 2$ & $-0.0015(2)$ & $-0.06791(19)$ & $0.65385(11)$ & 0.0393 & \\
\hline $\mathrm{C} 4$ & 0.30668 (19) & $0.18923(19)$ & $0.71322(11)$ & $0.0376(3)$ & \\
\hline $\mathrm{C} 5$ & $0.32002(19)$ & $0.11774(19)$ & $0.82021(11)$ & $0.0368(3)$ & \\
\hline C6 & $0.1393(2)$ & -0.04093 (19) & $0.83867(11)$ & $0.0383(3)$ & \\
\hline $\mathrm{C} 7$ & $0.3687(2)$ & $0.2764(2)$ & $0.90965(12)$ & $0.0460(4)$ & \\
\hline $\mathrm{H} 7 \mathrm{~A}$ & 0.3993 & 0.2375 & 0.9775 & $0.055^{*}$ & \\
\hline H7B & 0.4802 & 0.3803 & 0.8970 & $0.055^{*}$ & \\
\hline $\mathrm{C} 8$ & $0.2092(2)$ & $0.3359(2)$ & $0.91640(13)$ & $0.0527(4)$ & \\
\hline $\mathrm{H} 8$ & 0.1068 & 0.2623 & 0.9479 & $0.063 *$ & \\
\hline C9 & $0.2045(3)$ & $0.4828(3)$ & $0.88144(18)$ & $0.0759(6)$ & \\
\hline H9A & 0.3047 & 0.5594 & 0.8496 & $0.091^{*}$ & \\
\hline H9B & 0.1010 & 0.5118 & 0.8882 & $0.091^{*}$ & \\
\hline $\mathrm{C} 10$ & $0.4766(2)$ & $0.0383(2)$ & $0.82568(12)$ & $0.0463(4)$ & \\
\hline H10 & 0.4680 & -0.0253 & 0.8897 & $0.057(5)^{*}$ & \\
\hline C11 & $0.4322(2)$ & $-0.1006(2)$ & $0.73331(14)$ & $0.0510(4)$ & \\
\hline $\mathrm{C} 12$ & $0.3973(3)$ & $-0.2041(3)$ & $0.65519(17)$ & $0.0620(5)$ & \\
\hline $\mathrm{C} 13$ & $0.3521(4)$ & $-0.3299(3)$ & $0.55608(19)$ & $0.0882(7)$ & \\
\hline $\mathrm{H} 13 \mathrm{~A}$ & 0.4699 & -0.3204 & 0.5319 & $0.106^{*}$ & \\
\hline H13B & 0.2858 & -0.4551 & 0.5716 & $0.106^{*}$ & \\
\hline C14 & $0.2356(5)$ & $-0.2930(4)$ & $0.4700(2)$ & $0.1143(10)$ & \\
\hline H14A & 0.2091 & -0.3809 & 0.4086 & $0.171^{*}$ & \\
\hline H14B & 0.3027 & -0.1714 & 0.4518 & $0.171^{*}$ & \\
\hline $\mathrm{H} 14 \mathrm{C}$ & 0.1185 & -0.3025 & 0.4931 & $0.171^{*}$ & \\
\hline $\mathrm{C} 15$ & $0.6801(2)$ & $0.1819(3)$ & $0.83485(16)$ & $0.0640(5)$ & \\
\hline H15A & 0.7656 & 0.1217 & 0.8353 & $0.096^{*}$ & \\
\hline
\end{tabular}




\begin{tabular}{lllll}
\hline H15B & 0.7106 & 0.2628 & 0.8999 & $0.096^{*}$ \\
H15C & 0.6922 & 0.2517 & 0.7751 & $0.096^{*}$ \\
\hline
\end{tabular}

Atomic displacement parameters $\left(\AA^{2}\right)$

\begin{tabular}{lllllll}
\hline & $U^{11}$ & $U^{22}$ & $U^{33}$ & $U^{12}$ & $U^{13}$ & $U^{23}$ \\
\hline $\mathrm{N} 1$ & $0.0343(6)$ & $0.0402(6)$ & $0.0336(6)$ & $0.0085(5)$ & $0.0022(5)$ & $0.0091(5)$ \\
$\mathrm{N} 3$ & $0.0409(7)$ & $0.0458(7)$ & $0.0270(6)$ & $0.0083(5)$ & $-0.0027(5)$ & $0.0108(5)$ \\
O2 & $0.0463(6)$ & $0.0661(7)$ & $0.0372(6)$ & $-0.0001(5)$ & $-0.0087(5)$ & $0.0094(5)$ \\
O4 & $0.0494(6)$ & $0.0501(6)$ & $0.0469(7)$ & $0.0019(5)$ & $0.0011(5)$ & $0.0168(5)$ \\
O6 & $0.0592(7)$ & $0.0696(8)$ & $0.0345(6)$ & $0.0109(6)$ & $0.0028(5)$ & $0.0210(5)$ \\
C1 & $0.0428(8)$ & $0.0541(9)$ & $0.0541(10)$ & $0.0037(7)$ & $0.0054(7)$ & $0.0182(8)$ \\
C2 & $0.0366(7)$ & $0.0430(8)$ & $0.0328(8)$ & $0.0117(6)$ & $-0.0010(6)$ & $0.0069(6)$ \\
C4 & $0.0357(7)$ & $0.0389(7)$ & $0.0326(7)$ & $0.0100(6)$ & $0.0014(6)$ & $0.0072(6)$ \\
C5 & $0.0351(7)$ & $0.0424(7)$ & $0.0276(7)$ & $0.0115(6)$ & $-0.0017(5)$ & $0.0054(5)$ \\
C6 & $0.0396(7)$ & $0.0429(8)$ & $0.0302(7)$ & $0.0143(6)$ & $0.0026(6)$ & $0.0081(6)$ \\
C7 & $0.0433(8)$ & $0.0508(9)$ & $0.0329(8)$ & $0.0110(7)$ & $-0.0046(6)$ & $-0.0011(6)$ \\
C8 & $0.0504(9)$ & $0.0550(10)$ & $0.0433(9)$ & $0.0138(8)$ & $0.0031(7)$ & $-0.0034(7)$ \\
C9 & $0.0711(13)$ & $0.0635(12)$ & $0.0904(16)$ & $0.0273(10)$ & $0.0032(11)$ & $0.0041(11)$ \\
C10 & $0.0434(8)$ & $0.0571(9)$ & $0.0387(8)$ & $0.0215(7)$ & $-0.0007(6)$ & $0.0112(7)$ \\
C11 & $0.0491(9)$ & $0.0562(10)$ & $0.0533(10)$ & $0.0268(8)$ & $0.0064(8)$ & $0.0122(8)$ \\
C12 & $0.0633(11)$ & $0.0639(11)$ & $0.0663(12)$ & $0.0352(9)$ & $0.0044(9)$ & $0.0051(9)$ \\
C13 & $0.1023(18)$ & $0.0897(16)$ & $0.0816(16)$ & $0.0565(14)$ & $-0.0012(14)$ & $-0.0187(13)$ \\
C14 & $0.146(3)$ & $0.130(2)$ & $0.0667(16)$ & $0.064(2)$ & $0.0027(17)$ & $-0.0222(15)$ \\
C15 & $0.0396(9)$ & $0.0777(12)$ & $0.0671(12)$ & $0.0194(9)$ & $-0.0034(8)$ & $0.0029(10)$ \\
& & & & & & \\
\hline
\end{tabular}

Geometric parameters $\left(\AA,{ }^{\circ}\right)$

\begin{tabular}{|c|c|c|c|}
\hline $\mathrm{N} 1-\mathrm{C} 2$ & $1.3800(18)$ & C7-H7A & 0.9700 \\
\hline $\mathrm{N} 1-\mathrm{C} 6$ & 1.3804 (17) & $\mathrm{C} 7-\mathrm{H} 7 \mathrm{~B}$ & 0.9700 \\
\hline $\mathrm{N} 1-\mathrm{C} 1$ & $1.4687(18)$ & $\mathrm{C} 8-\mathrm{C} 9$ & $1.292(3)$ \\
\hline $\mathrm{N} 3-\mathrm{C} 2$ & $1.3648(18)$ & $\mathrm{C} 8-\mathrm{H} 8$ & 0.9300 \\
\hline $\mathrm{N} 3-\mathrm{C} 4$ & $1.3701(17)$ & $\mathrm{C} 9-\mathrm{H} 9 \mathrm{~A}$ & 0.9300 \\
\hline $\mathrm{N} 3-\mathrm{H} 3$ & $0.85(2)$ & C9-H9B & 0.9300 \\
\hline $\mathrm{O} 2-\mathrm{C} 2$ & $1.2140(16)$ & $\mathrm{C} 10-\mathrm{C} 11$ & $1.471(2)$ \\
\hline $\mathrm{O} 4-\mathrm{C} 4$ & $1.2032(17)$ & $\mathrm{C} 10-\mathrm{C} 15$ & $1.526(2)$ \\
\hline $\mathrm{O} 6-\mathrm{C} 6$ & $1.2083(17)$ & $\mathrm{C} 10-\mathrm{H} 10$ & 0.9800 \\
\hline $\mathrm{C} 1-\mathrm{H} 1 \mathrm{~A}$ & 0.9600 & $\mathrm{C} 11-\mathrm{C} 12$ & $1.182(2)$ \\
\hline $\mathrm{C} 1-\mathrm{H} 1 \mathrm{~B}$ & 0.9600 & $\mathrm{C} 12-\mathrm{C} 13$ & $1.474(3)$ \\
\hline $\mathrm{C} 1-\mathrm{H} 1 \mathrm{C}$ & 0.9600 & $\mathrm{C} 13-\mathrm{C} 14$ & $1.460(3)$ \\
\hline $\mathrm{C} 1-\mathrm{H} 1 \mathrm{D}$ & 0.9600 & $\mathrm{C} 13-\mathrm{H} 13 \mathrm{~A}$ & 0.9700 \\
\hline $\mathrm{C} 1-\mathrm{H} 1 \mathrm{E}$ & 0.9600 & C13-H13B & 0.9700 \\
\hline $\mathrm{C} 1-\mathrm{H} 1 \mathrm{~F}$ & 0.9600 & $\mathrm{C} 14-\mathrm{H} 14 \mathrm{~A}$ & 0.9600 \\
\hline $\mathrm{C} 4-\mathrm{C} 5$ & $1.5151(18)$ & $\mathrm{C} 14-\mathrm{H} 14 \mathrm{~B}$ & 0.9600 \\
\hline $\mathrm{C} 5-\mathrm{C} 6$ & 1.5248 (19) & $\mathrm{C} 14-\mathrm{H} 14 \mathrm{C}$ & 0.9600 \\
\hline $\mathrm{C} 5-\mathrm{C} 7$ & $1.541(2)$ & $\mathrm{C} 15-\mathrm{H} 15 \mathrm{~A}$ & 0.9600 \\
\hline $\mathrm{C} 5-\mathrm{C} 10$ & $1.574(2)$ & $\mathrm{C} 15-\mathrm{H} 15 \mathrm{~B}$ & 0.9600 \\
\hline $\mathrm{C} 7-\mathrm{C} 8$ & $1.498(2)$ & $\mathrm{C} 15-\mathrm{H} 15 \mathrm{C}$ & 0.9600 \\
\hline
\end{tabular}




\begin{tabular}{|c|c|c|c|}
\hline $\mathrm{C} 2-\mathrm{N} 1-\mathrm{C} 6$ & $123.81(12)$ & $\mathrm{O} 6-\mathrm{C} 6-\mathrm{C} 5$ & $120.29(12)$ \\
\hline $\mathrm{C} 2-\mathrm{N} 1-\mathrm{C} 1$ & $117.84(12)$ & $\mathrm{N} 1-\mathrm{C} 6-\mathrm{C} 5$ & $119.14(12)$ \\
\hline $\mathrm{C} 6-\mathrm{N} 1-\mathrm{C} 1$ & $118.24(12)$ & $\mathrm{C} 8-\mathrm{C} 7-\mathrm{C} 5$ & $112.61(12)$ \\
\hline $\mathrm{C} 2-\mathrm{N} 3-\mathrm{C} 4$ & $127.42(12)$ & $\mathrm{C} 8-\mathrm{C} 7-\mathrm{H} 7 \mathrm{~A}$ & 109.1 \\
\hline $\mathrm{C} 2-\mathrm{N} 3-\mathrm{H} 3$ & $113.2(12)$ & $\mathrm{C} 5-\mathrm{C} 7-\mathrm{H} 7 \mathrm{~A}$ & 109.1 \\
\hline $\mathrm{C} 4-\mathrm{N} 3-\mathrm{H} 3$ & $119.3(12)$ & $\mathrm{C} 8-\mathrm{C} 7-\mathrm{H} 7 \mathrm{~B}$ & 109.1 \\
\hline $\mathrm{N} 1-\mathrm{C} 1-\mathrm{H} 1 \mathrm{~A}$ & 109.5 & $\mathrm{C} 5-\mathrm{C} 7-\mathrm{H} 7 \mathrm{~B}$ & 109.1 \\
\hline $\mathrm{N} 1-\mathrm{C} 1-\mathrm{H} 1 \mathrm{~B}$ & 109.5 & $\mathrm{H} 7 \mathrm{~A}-\mathrm{C} 7-\mathrm{H} 7 \mathrm{~B}$ & 107.8 \\
\hline $\mathrm{H} 1 \mathrm{~A}-\mathrm{C} 1-\mathrm{H} 1 \mathrm{~B}$ & 109.5 & $\mathrm{C} 9-\mathrm{C} 8-\mathrm{C} 7$ & $124.36(18)$ \\
\hline $\mathrm{N} 1-\mathrm{C} 1-\mathrm{H} 1 \mathrm{C}$ & 109.5 & $\mathrm{C} 9-\mathrm{C} 8-\mathrm{H} 8$ & 117.8 \\
\hline $\mathrm{H} 1 \mathrm{~A}-\mathrm{C} 1-\mathrm{H} 1 \mathrm{C}$ & 109.5 & $\mathrm{C} 7-\mathrm{C} 8-\mathrm{H} 8$ & 117.8 \\
\hline $\mathrm{H} 1 \mathrm{~B}-\mathrm{C} 1-\mathrm{H} 1 \mathrm{C}$ & 109.5 & $\mathrm{C} 8-\mathrm{C} 9-\mathrm{H} 9 \mathrm{~A}$ & 120.0 \\
\hline $\mathrm{N} 1-\mathrm{C} 1-\mathrm{H} 1 \mathrm{D}$ & 109.5 & $\mathrm{C} 8-\mathrm{C} 9-\mathrm{H} 9 \mathrm{~B}$ & 120.0 \\
\hline $\mathrm{H} 1 \mathrm{~A}-\mathrm{C} 1-\mathrm{H} 1 \mathrm{D}$ & 141.1 & $\mathrm{H} 9 \mathrm{~A}-\mathrm{C} 9-\mathrm{H} 9 \mathrm{~B}$ & 120.0 \\
\hline $\mathrm{H} 1 \mathrm{~B}-\mathrm{C} 1-\mathrm{H} 1 \mathrm{D}$ & 56.3 & $\mathrm{C} 11-\mathrm{C} 10-\mathrm{C} 15$ & $110.95(15)$ \\
\hline $\mathrm{H} 1 \mathrm{C}-\mathrm{C} 1-\mathrm{H} 1 \mathrm{D}$ & 56.3 & $\mathrm{C} 11-\mathrm{C} 10-\mathrm{C} 5$ & $109.25(12)$ \\
\hline $\mathrm{N} 1-\mathrm{C} 1-\mathrm{H} 1 \mathrm{E}$ & 109.5 & $\mathrm{C} 15-\mathrm{C} 10-\mathrm{C} 5$ & $114.96(14)$ \\
\hline $\mathrm{H} 1 \mathrm{~A}-\mathrm{C} 1-\mathrm{H} 1 \mathrm{E}$ & 56.3 & $\mathrm{C} 11-\mathrm{C} 10-\mathrm{H} 10$ & 107.1 \\
\hline $\mathrm{H} 1 \mathrm{~B}-\mathrm{C} 1-\mathrm{H} 1 \mathrm{E}$ & 141.1 & $\mathrm{C} 15-\mathrm{C} 10-\mathrm{H} 10$ & 107.1 \\
\hline $\mathrm{H} 1 \mathrm{C}-\mathrm{C} 1-\mathrm{H} 1 \mathrm{E}$ & 56.3 & $\mathrm{C} 5-\mathrm{C} 10-\mathrm{H} 10$ & 107.1 \\
\hline $\mathrm{H} 1 \mathrm{D}-\mathrm{C} 1-\mathrm{H} 1 \mathrm{E}$ & 109.5 & $\mathrm{C} 12-\mathrm{C} 11-\mathrm{C} 10$ & $176.00(18)$ \\
\hline $\mathrm{N} 1-\mathrm{C} 1-\mathrm{H} 1 \mathrm{~F}$ & 109.5 & $\mathrm{C} 11-\mathrm{C} 12-\mathrm{C} 13$ & $178.4(2)$ \\
\hline $\mathrm{H} 1 \mathrm{~A}-\mathrm{C} 1-\mathrm{H} 1 \mathrm{~F}$ & 56.3 & $\mathrm{C} 14-\mathrm{C} 13-\mathrm{C} 12$ & $113.7(2)$ \\
\hline $\mathrm{H} 1 \mathrm{~B}-\mathrm{C} 1-\mathrm{H} 1 \mathrm{~F}$ & 56.3 & $\mathrm{C} 14-\mathrm{C} 13-\mathrm{H} 13 \mathrm{~A}$ & 108.8 \\
\hline $\mathrm{H} 1 \mathrm{C}-\mathrm{C} 1-\mathrm{H} 1 \mathrm{~F}$ & 141.1 & $\mathrm{C} 12-\mathrm{C} 13-\mathrm{H} 13 \mathrm{~A}$ & 108.8 \\
\hline $\mathrm{H} 1 \mathrm{D}-\mathrm{C} 1-\mathrm{H} 1 \mathrm{~F}$ & 109.5 & $\mathrm{C} 14-\mathrm{C} 13-\mathrm{H} 13 \mathrm{~B}$ & 108.8 \\
\hline $\mathrm{H} 1 \mathrm{E}-\mathrm{C} 1-\mathrm{H} 1 \mathrm{~F}$ & 109.5 & $\mathrm{C} 12-\mathrm{C} 13-\mathrm{H} 13 \mathrm{~B}$ & 108.8 \\
\hline $\mathrm{O} 2-\mathrm{C} 2-\mathrm{N} 3$ & $121.50(13)$ & $\mathrm{H} 13 \mathrm{~A}-\mathrm{C} 13-\mathrm{H} 13 \mathrm{~B}$ & 107.7 \\
\hline $\mathrm{O} 2-\mathrm{C} 2-\mathrm{N} 1$ & $121.22(13)$ & $\mathrm{C} 13-\mathrm{C} 14-\mathrm{H} 14 \mathrm{~A}$ & 109.5 \\
\hline $\mathrm{N} 3-\mathrm{C} 2-\mathrm{N} 1$ & $117.27(12)$ & $\mathrm{C} 13-\mathrm{C} 14-\mathrm{H} 14 \mathrm{~B}$ & 109.5 \\
\hline $\mathrm{O} 4-\mathrm{C} 4-\mathrm{N} 3$ & $120.57(12)$ & $\mathrm{H} 14 \mathrm{~A}-\mathrm{C} 14-\mathrm{H} 14 \mathrm{~B}$ & 109.5 \\
\hline $\mathrm{O} 4-\mathrm{C} 4-\mathrm{C} 5$ & $122.59(12)$ & $\mathrm{C} 13-\mathrm{C} 14-\mathrm{H} 14 \mathrm{C}$ & 109.5 \\
\hline $\mathrm{N} 3-\mathrm{C} 4-\mathrm{C} 5$ & $116.82(12)$ & $\mathrm{H} 14 \mathrm{~A}-\mathrm{C} 14-\mathrm{H} 14 \mathrm{C}$ & 109.5 \\
\hline $\mathrm{C} 4-\mathrm{C} 5-\mathrm{C} 6$ & $114.41(11)$ & $\mathrm{H} 14 \mathrm{~B}-\mathrm{C} 14-\mathrm{H} 14 \mathrm{C}$ & 109.5 \\
\hline $\mathrm{C} 4-\mathrm{C} 5-\mathrm{C} 7$ & $108.82(12)$ & $\mathrm{C} 10-\mathrm{C} 15-\mathrm{H} 15 \mathrm{~A}$ & 109.5 \\
\hline $\mathrm{C} 6-\mathrm{C} 5-\mathrm{C} 7$ & $108.29(12)$ & $\mathrm{C} 10-\mathrm{C} 15-\mathrm{H} 15 \mathrm{~B}$ & 109.5 \\
\hline $\mathrm{C} 4-\mathrm{C} 5-\mathrm{C} 10$ & $108.59(12)$ & $\mathrm{H} 15 \mathrm{~A}-\mathrm{C} 15-\mathrm{H} 15 \mathrm{~B}$ & 109.5 \\
\hline $\mathrm{C} 6-\mathrm{C} 5-\mathrm{C} 10$ & $105.19(11)$ & $\mathrm{C} 10-\mathrm{C} 15-\mathrm{H} 15 \mathrm{C}$ & 109.5 \\
\hline $\mathrm{C} 7-\mathrm{C} 5-\mathrm{C} 10$ & $111.55(11)$ & $\mathrm{H} 15 \mathrm{~A}-\mathrm{C} 15-\mathrm{H} 15 \mathrm{C}$ & 109.5 \\
\hline $\mathrm{O} 6-\mathrm{C} 6-\mathrm{N} 1$ & $120.54(13)$ & $\mathrm{H} 15 \mathrm{~B}-\mathrm{C} 15-\mathrm{H} 15 \mathrm{C}$ & 109.5 \\
\hline $\mathrm{C} 10-\mathrm{C} 5-\mathrm{C} 7-\mathrm{C} 8$ & $-171.51(13)$ & $\mathrm{C} 5-\mathrm{C} 7-\mathrm{C} 8-\mathrm{C} 9$ & $-103.3(2)$ \\
\hline
\end{tabular}

Hydrogen-bond geometry $\left(A,{ }^{\circ}\right)$

$D-\mathrm{H} \cdots A \quad D-\mathrm{H} \quad \mathrm{H} \cdots A \quad D \cdots A \quad D-\mathrm{H} \cdots A$


Symmetry code: (i) $-x,-y,-z+1$. 